\title{
Effect of Local Steroid Injection for Acute Anterior Talofibular Ligament Partial Tear: A Randomized Trial
}

Chung-Ting Liu

Mackay Memorial Hospital

Ten-Fang Yang ( $\nabla$ tim09001099@gmail.com )

National Chiao Tung University College of Biological Science and Technology

Wei-Cheng Chen

Mackay Medical College

Research article

Keywords: Steroid, local injection, anterior talofibular ligament, partial tear

Posted Date: August 11th, 2020

DOI: https://doi.org/10.21203/rs.3.rs-42571/v1

License: (1) (i) This work is licensed under a Creative Commons Attribution 4.0 International License.

Read Full License 


\section{Abstract}

\section{Background:}

The symptoms of acute ankle anterior talofibular ligament (ATFL) partial tear includes pain, swelling, and range of motion limitation. Local steroid injection can provide strong anti-inflammatory effect. The aim of the study is to evaluate the effectiveness and complications of local steroid injection for acute ATFL partial tear in short term.

\section{Materials and Methods:}

This is a prospective randomized study. Forty patients with acute isolated ATFL partial tear diagnosed by sonography were equally separated into two groups. The steroid group received 1cc Diprospan local injection whereas the control group received $1 \mathrm{cc}$ normal saline injection. We used sonography to check the tendon rupture status before injection, 6 weeks and 12 weeks after injection. We also used American Orthopaedic Foot \& Ankle Society (AOFAS) score and Pain Visual Analogue Scale (VAS) score to evaluate the function and pain before injection, 1 week, 3 weeks, 6 weeks, and 12 weeks after injection. Student $t$ test was used to compare the statistically significant difference between groups.

\section{Result:}

There was no deteriorating of ATFL tear found by sonography in both groups until 12 weeks. The average AOFAS score was higher than 80 after 1 weeks in steroid group while the score was higher than 80 after 3 weeks in control group. AOFAS score was significantly higher in steroid group for the first 3 weeks after injection ( $p=3.2 \times 10^{-7}$ and 0.022 , at 1 week and 3 weeks respectively), but there was no significant difference between groups afterward. VAS score was significant higher in steroid group only in the first week $(p=0.0073)$ and there was no significant difference between groups afterward

\section{Conclusion:}

Steroid local injection can safely shorten the period of functional disability and relieve pain for acute ATFL partial tear in short term.

\section{Trial registration:}

ChiCTR, ChiCTR2000029629, registered 07 February 2020

http://www.chictr.org.cn/showproj.aspx?proj=49198

\section{Background}


Anterior talofibular ligament (ATFL) is the most common injured structure in ankle joint. It is approximately $2 \mathrm{~mm}$ thick, $7 \mathrm{~mm}$ wide, and $10 \mathrm{~mm}$ long. Its main function is to resist inversion and plantarflexion. Approximately two-thirds of ankle sprains tend to be isolated injuries to the ATFL which is the weakest ligament in the lateral collateral complex of the ankle (1-3). The occurrence of combined rupture of the ATFL and calcaneofibular ligament (CFL) is reported as $20 \%$, and the posterior talofibular ligament (PTFL) injury does not occur unless the ankle joint undergoes dislocation (4).

The severity of Ligament injury is defined by American Medical Association (AMA) classification (5). Grade I reveals stretching with no gross disruption of ligament fibers. Grade II reveals moderate, partial tear of ligament without complete disruption. And grade III reveals severe, complete tear of ligament.

Physical examination for ATFL injury is ankle anterior drawer test (6). It helps to identify excessive anterior displacement of the talus on the tibia. Ultrasound can be a valuable instrument for ATFL injury, but it is operator-dependent (7). X-ray radiography is also necessary to rule out any other bony fractures. If the patient experiences more than one injuries or has ankle instability, Magnetic Resonance Imaging (MRI) may be indicated (8).

The symptoms of ATFL injury includes pain, swelling, weakness, and instability of ankle joint. Most of the patients with acute isolated ATFL injury can be treated conservatively. The managements include protection, rest, ice packing, compression, and elevation for the first 3 days. Thereafter, rehabilitation program should be started as soon as possible. In the acute inflammatory stage of the ankle injuries, nonsteroidal anti-inflammatory medications (NSAID) can be used to lessen the severity of symptoms. It is also recommended to wrap the ankle with an elastic bandage to control the edema (9). Patients can be provided with crutches to facilitate mobility when the ankle sprain is painful, but weight-bearing is possible if tolerated.

Steroid injection for the injured structure is still controversial. Steroid has a strong effect of antiinflammation and it may improve the symptoms of ATFL injury. Steroid injection for shoulder rotator cuff tendon rupture was proved to be safe and effective in short term to relieve pain in a prospective randomized study by this research group (10). However, in-vitro studies have been shown that steroids can induce necrosis on fibroblasts and tenocytes so that it has a negative effect for tissue healing (11). This mandate further research to clarify.

In this study, steroid local injection will be used for the isolated acute AMA grade II ATFL injury. Most ATFL partial tear heals with conservative treatment in 6 to 12 weeks, but painful disability is noted before completely recovering (12). The aim of the study is to evaluate the effectiveness and complications of local steroid injection for acute ATFL partial tear in short term.

\section{Materials And Methods}

This prospective randomized study was approved by the ethics committee in our hospital and clinical trial registration center of World Health Organization (WHO). This study adhered to CONSORT guidelines. 
The principles and laws of clinical trials were followed. All participants in this study had to sign the informed consent.

We enrolled patients diagnosed with acute ATFL injury. Inclusion criteria were isolated ATFL partial tear (AMA grade II injury) diagnosed by sonography and injury less than 3 days. Exclusion criteria were 1 . recurrent ATFL injury, 2. existing other chronic lesion of ankle joint, 3 . athlete, 4 . age less than 20 or more than 50 years old.

All of the participants were recruited from Orthopedics department of Mackay Memorial Hospital, Taipei. Forty enrolled patients were divided into two equal groups by block randomization. This method is designed to randomize subjects into two equal groups with balance in sample size. It prevents the selection bias by randomization and balance the accidental bias. It produces the comparable groups and eliminates the source of bias in treatment assignments (13). Patients in steroid group received ATFL local injection with $1 \mathrm{cc}$ Diprospan which contains betamethasone disodium phosphate $2 \mathrm{mg}$ and betamethasone dipropionate $5 \mathrm{mg}$. While patients in the control group received an injection with $1 \mathrm{cc}$ normal saline. Twenty patients in each group were recruited. This was a single blind study in which patients were not aware of what regimen they received. Local injection was done and guided with echo by a single experienced orthopedic surgeon. After the injection, ankle wrapping was applied with an elastic bandage to control the edema. Weight-bearing was allowed if tolerated.

Sonography was performed before the injection, 6 weeks, and 12 weeks after the injection. All sonography examinations were performed by a single experienced orthopedic surgeon.

Pain Visual Analogue Scale (VAS) score and American Orthopaedic Foot \& Ankle Society (AOFAS) AnkleHindfoot Score were both measured and compared between the two groups before the injection, and at 1 week, 3 weeks, 6 weeks and 12 weeks after the injection $(14,15)$. AOFAS score is the most commonly used scores for evaluating the severity of ankle and foot injury. It combines both clinician-reported and patient-reported parts. There are total 100 points and higher scores indicates better function.

The AOFAS scores and VAS scores between groups were compared with statistical analysis. We used Shapiro-Wilk test to evaluate the normality of sample data distribution and student $t$ test was applied to compare the scores between groups. A $p$ value less than 0.05 was regarded as statistically significant different.

Physical examination of ankle anterior drawer test was performed at 12 weeks for each patient to evaluate the ankle instability (6).

\section{Results}

The average age was 26.5 years old in steroid group and 31.0 in control group. Other baseline demographic and clinical characteristics were showed in Table 1. 
Table 1

\begin{tabular}{|lll|}
\hline & Steroid group & Control group \\
\hline Average age & 26.5 & 31.0 \\
\hline Male : Female & $8: 12$ & $11: 9$ \\
\hline Right ankle : Left ankle & $6: 14$ & $7: 13$ \\
\hline Average BMI & 23.4 & 24.5 \\
\hline
\end{tabular}

There was no deteriorating of ATFL tear found by sonography in both groups until 12 weeks.

Before injection, the average AOFAS score was 55.1 (range, 31 to 71) in steroid group and 55.4 (range, 34 to 71) in control group with no significant difference between groups $(p=0.96)$. One week after injection, it was 83.5 (range, 77 to 87 ) in steroid group and 69.8 (range, 65 to 81) in control group with significant difference between groups $\left(p=3.2 \times 10^{-7}\right)$. Three weeks after injection, it was 86.9 (range, 80 to 90 ) in steroid group and 82.8 (range, 81 to 87 ) in control group with significant difference between groups ( $p=$ 0.022). Six weeks after injection, it was 91.9 (range, 87 to 100) in steroid group and 87.2 (range, 81 to 90 ) in control group without significant difference between groups $(p=0.27)$. Twelve weeks after injection, it was 95.3 (range, 90 to 100) in steroid group and 93.7 (range, 90 to 97) in control group without significant difference between groups $(p=0.37)$. The average AOFAS score was higher than 80 after 1 weeks in steroid group while the score was higher than 80 after 3 weeks in control group. AOFAS score was significantly higher in steroid group for the 3 weeks after injection, but there was no significant difference between groups afterward (Fig. 1).

Before injection, the average VAS score was 7.27 (range, 6 to 9) in steroid group and 7.18 (range, 6 to 9 ) in control group with no significant difference between groups $(p=0.83)$. One week after injection, it was 3.72 (range, 3 to 5 ) in steroid group and 4.73 (range, 3 to 6 ) in control group with significant difference between groups $(p=0.0073)$. Three weeks after injection, it was 2.55 (range, 1 to 4$)$ in steroid group and 3.18 (range, 2 to 5 ) in control group without significant difference between groups $(p=0.07)$. Six weeks after injection, it was 1.73 (range, 1 to 3 ) in steroid group and 2.00 (range, 1 to 4 ) in control group without significant difference between groups $(p=0.42)$. Twelve weeks after injection, it was 1.27 (range, 1 to 2$)$ in steroid group and 1.09 (range, 2 to 2 ) in control group without significant difference between groups ( $p$ $=0.29$ ). VAS score was significant higher in steroid group only in the first week and there was no significant difference between groups afterward (Fig. 2).

Ankle anterior drawer test was performed at 12 weeks after injection and revealed no ankle instability by this physical examination in all cases. No other complications were noted after injection in both groups until 12 weeks.

Steroid local injection can safely shorten the period of functional disability and relieve pain for acute ATFL partial tear in short term. 


\section{Discussions}

The symptoms of acute ATFL partial tear including pain, swelling, weakness, and range of motion limitation. Most of the patients can recover in a few weeks with conservative treatment. The inflammation stage takes the first 3 to 7 days which is the most uncomfortable. The painful disability at the first week has significant negative effects on working and quality of life. Steroid has a strong effect of anti-inflammation and it may improve the symptoms of injury at acute stage. Steroid injection for rotator cuff tendon rupture was proved to be safe and effective in short term to relieve pain in a prospective randomized study previously done by our team (10). However, in-vitro studies have shown steroids have necrosis-inducing effects on fibroblasts and tenocytes so that it may have a negative effect for tissue healing (11). Although steroid injection is very popular in clinical practice, it is still controversial for the injured tendons and ligaments. In the current study, we revealed steroid local injection can safely shorten the period of functional disability and relieve pain for acute ATFL partial tear.

Acute ankle ligament injuries are almost always managed by non-operative methods. Successful treatment can be achieved with individualized, aggressive and non-operative measures. A previous study revealed that patients who underwent acute surgical treatment took longer to return to work (16). Also, studies revealed that surgery was not helpful in reducing additional complications, costs and risks (17). Lateral ligament reconstruction of the ankle is indicated when conservative measures have failed to improve functional lateral ankle instability in order to prevent subsequent disorders such as osteochondral lesions of the talar dome and osteoarthritis of the ankle (18). The direct anatomic repair of lateral ligaments of the ankle, originally described by Broström (19), is popular and effective if indicated. The subsequent augmentation of the technique by additionally tightening the inferior extensor retinaculum has resulted in good outcomes in the literatures (20). However, the open technique requires at least a 4-cm-long incision with significant dissection and soft tissue debridement, and it sometimes causes superficial nerve injury (20). Recently, several authors have reported good results using an arthroscopy-assisted lateral ligament repair (21).

The clinical evidence of Platelet Rich Plasma (PRP) on soft tissue healing and repair is so far convincing. Case series have shown the beneficial effect on the partial thickness rotator cuff tear (22). PRP has been increasingly used for therapeutic applications in sports-related injuries, and is thought to stimulate tissue healing. Some studies supported PRP injection for ATFL injury as an alternative non-surgical treatment option with the potential to prevent the development of chronic ankle instability and post-traumatic ankle osteoarthritis (23). And the other study revealed the PRP group presented the highest reduction in pain and better functional scores than the control group at 8 weeks. However, the results of both groups were similar at the end of follow-up period (24). The role of PRP in ankle ATFL injury is still not clear. Therefore, further prospective randomized study for PRP injection is necessary.

ATFL injury has some associated injuries which cannot be evaluated by sonography, including osteochondroal lesion of talar dome and bone contusion. These associated injuries can only be diagnosed by MRI. In a study conducted on 261 ankle MRI scans of athletes who had acute ankle 
sprains, acute osteochondral lesions of the lateral talar dome were seen in 20 ankles (7.7\%). And talar bone contusion risk increased more than three times for ATFL complete ruptures (25). If these associated injuries happen, the functional recovery and the healing process will be different from pure ligamentous injury. In the current study, we did not take associated osteochondral lesion and bone contusion into consideration because MRI was not performed in every patient.

The inclusion criteria of current study was ATFL acute injury less than 3 days. MRI was not the choice of current study because it cannot be available immediately. The definite diagnosis of ATFL injury is arthroscopy which is an invasive procedure and it cannot be apply for all the patients. Ultrasound can be a valuable technique for ATFL injury, but it is operator-dependent (7). Using sonography to evaluate ATFL can have some errors, including inter-observer and intra-observer variability. All sonographic examinations in this study were performed by the same experienced orthopedic surgeon to reduce the inter-observer variability, but intra-observer variability may still exist. In addition, the healing of ruptured ATFL was difficult to identified or compared by sonography (26). We performed sonography before injection, 6 weeks, and 12 weeks after injection. The only information we got from follow-up sonography were the decreasing inflammation and no deterioration of partial tear after injection. Besides, there is high interpatient variability in the structure of ATFL (27). Therefore, the results from sonography was difficult to be compared between groups.

There were some other limitations in the current study. Our sample size was too small, and the duration of follow-up was too short. Block randomization was used because the case number was not sufficient enough to perform the age and sex match distribution between groups. The period of follow-up was only 12 weeks in current study. However, ankle chronic instability after ligament injury was diagnosed after 12 weeks. Although we use ankle anterior drawer test to evaluate the stability for all patients at 12 weeks, the specificity and sensitivity of this physical examination is moderate. According to the literature, the specificity for the anterior drawer test assessed by arthrography ranged from 67.3 to 93.9 and the sensitivity ranged from 12.0 to 89.0 (28). Unlike acute ankle sprain, chronic ankle instability might require surgical intervention (29). Therefore, long term follow-up for ATFL partial tear treated by steroid local injection is necessary to evaluate its long term safety. A larger sample size is also required.

\section{Conclusion}

Steroid local injection can safely shorten the period of functional disability and relieve pain for acute ATFL partial tear in short term.

\section{Abbreviations}

ATFL

Anterior talofibular ligament

CFL

Calcaneofibular ligament 
MRI

Magnetic Resonance Imaging

AMA

American Medical Association

NSAID

Nonsteroidal anti-inflammatory drugs

AOFAS

Orthopaedic Foot \& Ankle Society

VAS

Pain Visual Analogue Scale

WHO

World Health Organization

CONSORT

Consolidated standards of reporting trials

PRP

Platelet Rich Plasma

\section{Declarations}

\section{Ethics approval and consent to participate}

This study was approved by the ethics committee of Mackay Memorial Hospital, Taipei, Taiwan. The IRB approval number is 16MMHIS163 (since 2019-Dec-29) and the clinical trial registration number is ChiCTR2000029629. Written informed consent was obtained from all individual participants included in the study.

\section{Consent for publication}

Written consent for publication was obtained from all individual participants.

\section{Availability of data and material}

The datasets used and analysed during the current study are available from the corresponding author on reasonable request.

\section{Competing interests}

No potential conflict of interest is reported by the authors. 


\section{Funding}

Not applicable

\section{Authors' contributions}

LCT (first author) collected data, performed the analytic calculations, and wrote the manuscript.

YTF (corresponding author) conceived of the presented idea, developed the theory, and supervised the whole work.

CWC (second author) collected data, provided cases, and wrote the manuscript.

All authors have read and approved the final manuscript.

\section{Acknowledgements}

The authors wish to acknowledge the assistance provided by Hsinchu National Chiao Tung University and the department of orthopedics of Taipei Mackay Memorial Hospital.

\section{References}

1. Hertel J. Functional Anatomy, Pathomechanics, and Pathophysiology of Lateral Ankle Instability. J Athl Train. 2002;37(4):364-75.

2. Milner $\mathrm{CE}$, Soames RW. Anatomy of the collateral ligaments of the human ankle joint. Foot Ankle Int. 1998;19(11):757-60.

3. Golano P, Vega J, de Leeuw PA, Malagelada F, Manzanares MC, Gotzens V, et al. Anatomy of the ankle ligaments: a pictorial essay. Knee surgery, sports traumatology, arthroscopy: official journal of the ESSKA. 2010;18(5):557-69.

4. Vuurberg G, Hoorntje A, Wink LM, van der Doelen BFW, van den Bekerom MP, Dekker R, et al.

Diagnosis, treatment and prevention of ankle sprains: update of an evidence-based clinical guideline. Br J Sports Med. 2018;52(15):956.

5. Harper JD. Determining foot and ankle impairments by the AMA fifth edition guides. Foot Ankle Clin. 2002;7(2):291-303.

6. van Dijk CN, Lim LS, Bossuyt PM, Marti RK. Physical examination is sufficient for the diagnosis of sprained ankles. The Journal of bone joint surgery British volume. 1996;78(6):958-62.

7. Jacobson JA. Musculoskeletal sonography and MR imaging. A role for both imaging methods. Radiologic clinics of North America. 1999;37(4):713-35. 
8. Kaminski TW, Hertel J, Amendola N, Docherty CL, Dolan MG, Hopkins JT, et al. National Athletic Trainers' Association position statement: conservative management and prevention of ankle sprains in athletes. J Athl Train. 2013;48(4):528-45.

9. Wolfe MW, Uhl TL, Mattacola CG, McCluskey LC. Management of ankle sprains. Am Family Phys. 2001;63(1):93-104.

10. Liu CT, Yang TF. Intra-substance steroid injection for full-thickness supraspinatus tendon rupture. BMC Musculoskelet Disord. 2019;20(1):569.

11. Zhang AZ, Ficklscherer A, Gulecyuz MF, Paulus AC, Niethammer TR, Jansson V, et al. Cell Toxicity in Fibroblasts, Tenocytes, and Human Mesenchymal Stem Cells-A Comparison of Necrosis and Apoptosis-Inducing Ability in Ropivacaine, Bupivacaine, and Triamcinolone. Arthroscopy: the journal of arthroscopic related surgery : official publication of the Arthroscopy Association of North America the International Arthroscopy Association. 2017;33(4):840-8.

12. Al-Mohrej OA, Al-Kenani NS. Acute ankle sprain: conservative or surgical approach? EFORT open reviews. 2016;1(2):34-44.

13. Suresh K. An overview of randomization techniques: An unbiased assessment of outcome in clinical research. Journal of human reproductive sciences. 2011;4(1):8-11.

14. Kitaoka HB, Alexander IJ, Adelaar RS, Nunley JA, Myerson MS, Sanders M. Clinical rating systems for the ankle-hindfoot, midfoot, hallux, and lesser toes. Foot Ankle Int. 1994;15(7):349-53.

15. Gould D, Kelly D, Goldstone L, Gammon J. Examining the validity of pressure ulcer risk assessment scales: developing and using illustrated patient simulations to collect the data. Journal of clinical nursing. 2001;10(5):697-706.

16. Kerkhoffs GM, Handoll HH, de Bie R, Rowe BH, Struijs PA. Surgical versus conservative treatment for acute injuries of the lateral ligament complex of the ankle in adults. The Cochrane database of systematic reviews. 2007(2):Cd000380.

17. Shrier I. Treatment of lateral collateral ligament sprains of the ankle: a critical appraisal of the literature. Clinical journal of sport medicine: official journal of the Canadian Academy of Sport Medicine. 1995;5(3):187-95.

18. Takao M, Ochi M, Uchio Y, Naito K, Kono T, Oae K. Osteochondral lesions of the talar dome associated with trauma. Arthroscopy: the journal of arthroscopic related surgery : official publication of the Arthroscopy Association of North America the International Arthroscopy Association. 2003;19(10):1061-7.

19. Brostrom L. Sprained ankles. V. Treatment and prognosis in recent ligament ruptures. Acta chirurgica Scandinavica. 1966;132(5):537-50.

20. Karlsson J, Eriksson BI, Bergsten T, Rudholm O, Sward L. Comparison of two anatomic reconstructions for chronic lateral instability of the ankle joint. Am J Sports Med. 1997;25(1):48-53.

21. Vega J, Golano P, Pellegrino A, Rabat E, Pena F. All-inside arthroscopic lateral collateral ligament repair for ankle instability with a knotless suture anchor technique. Foot Ankle Int. 2013;34(12):1701-9. 
22. Zafarani Z, Mirzaee F, Guity M, Aslani H. Clinical Results of Platelet-Rich Plasma for Partial Thickness Rotator Cuff Tears: A Case Series. The archives of bone joint surgery. 2017;5(5):328-31.

23. Lai MWW, Sit RWS. Healing of Complete Tear of the Anterior Talofibular Ligament and Early Ankle Stabilization after Autologous Platelet Rich Plasma: a Case Report and Literature Review. The archives of bone joint surgery. 2018;6(2):146-9.

24. Blanco-Rivera J, Elizondo-Rodriguez J, Simental-Mendia M, Vilchez-Cavazos F, Pena-Martinez VM, Acosta-Olivo C. Treatment of lateral ankle sprain with platelet-rich plasma: A randomized clinical study. Foot and ankle surgery: official journal of the European Society of Foot and Ankle Surgeons. 2019.

25. Roemer FW, Jomaah N, Niu J, Almusa E, Roger B, D'Hooghe P, et al. Ligamentous Injuries and the Risk of Associated Tissue Damage in Acute Ankle Sprains in Athletes: A Cross-sectional MRI Study. Am J Sports Med. 2014;42(7):1549-57.

26. Hubbard TJ, Hicks-Little CA. Ankle ligament healing after an acute ankle sprain: an evidence-based approach. J Athl Train. 2008;43(5):523-9.

27. Milner CE, Soames RW. Anatomical variations of the anterior talofibular ligament of the human ankle joint. Journal of anatomy. 1997;191(Pt 3):457-8.

28. Schneiders $A$, Karas $S$. The accuracy of clinical tests in diagnosing ankle ligament injury. European Journal of Physiotherapy. 2016;18(4):245-53.

29. Al-Mohrej OA, Al-Kenani NS. Chronic ankle instability: Current perspectives. Avicenna journal of medicine. 2016;6(4):103-8.

\section{Figures}

\section{AOFAS score}

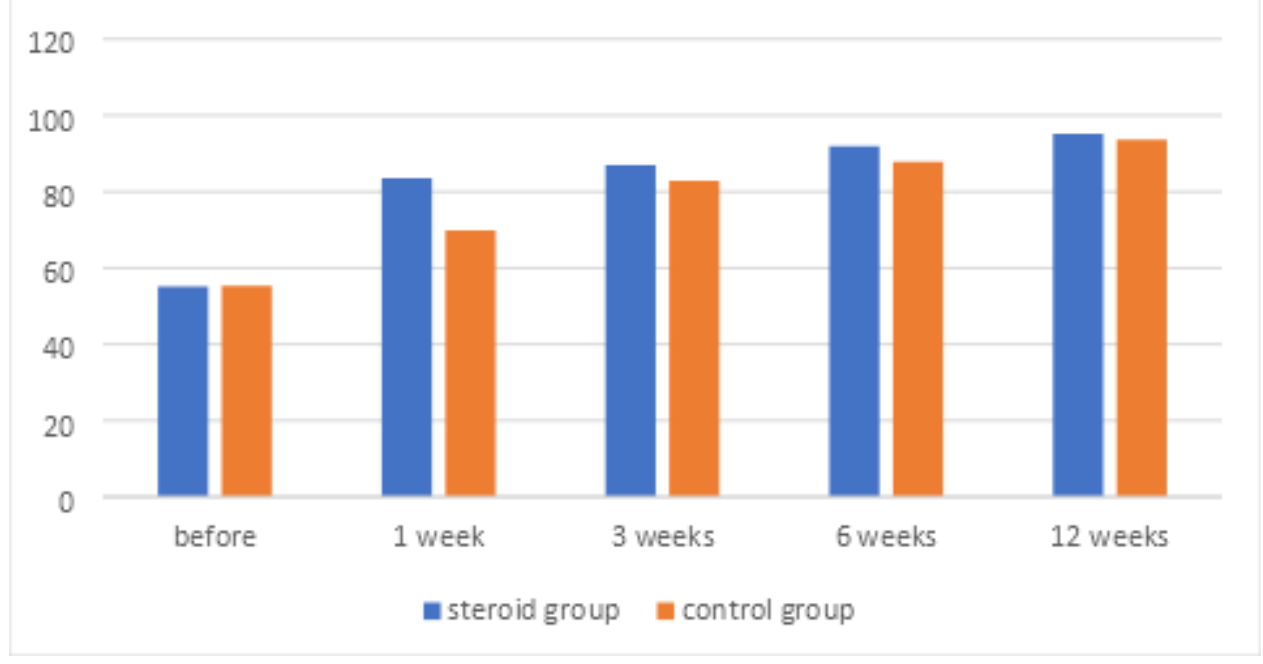

\section{Figure 1}


The average AOFAS score was higher than 80 after 1 weeks in steroid group while the score was higher than 80 after 3 weeks in control group. AOFAS score was significantly higher in steroid group for the first 3 weeks after injection, but there was no significant difference between groups afterward

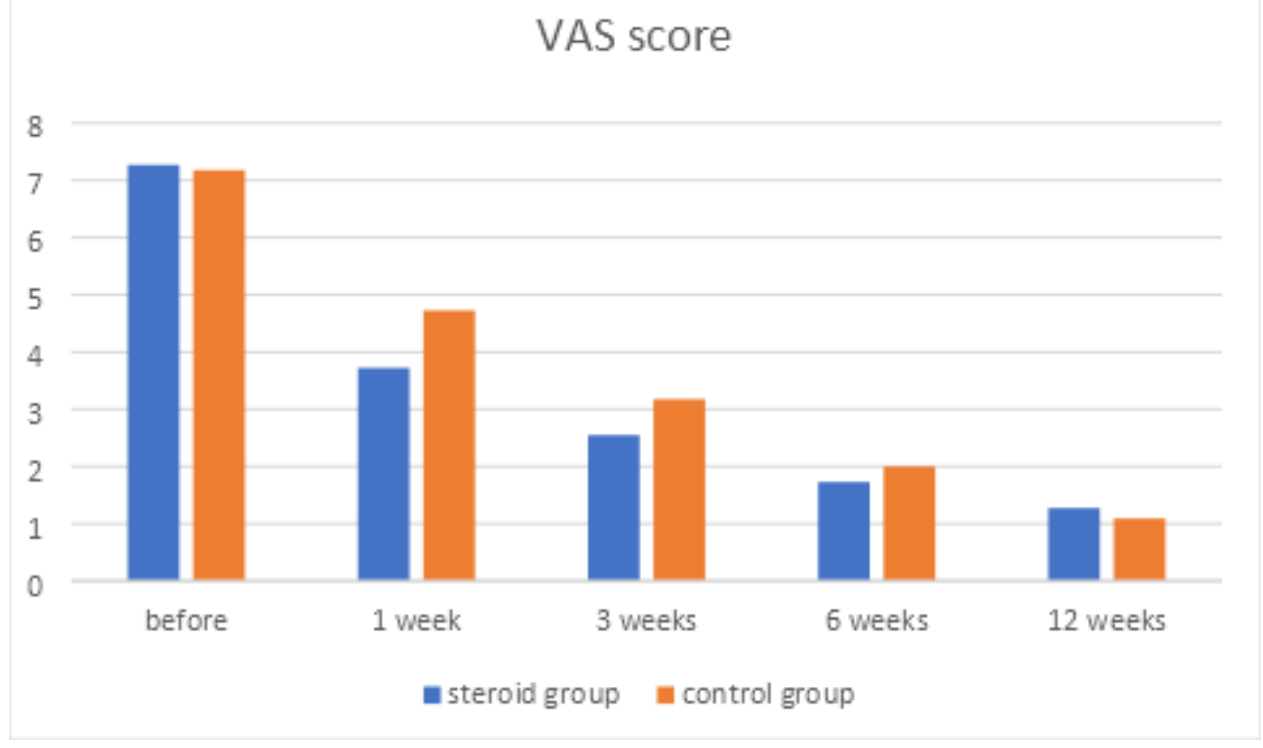

Figure 2

VAS score was significant higher in steroid group only in the first week and there was no significant difference between groups afterward

\section{Supplementary Files}

This is a list of supplementary files associated with this preprint. Click to download.

- CONSORTChecklist.doc 